\title{
Constrained Controllability of the $h$-Difference Fractional Control Systems with Caputo Type Operator
}

\author{
Ewa Pawluszewicz \\ Bialystok University of Technology, 15-351 Bialystok, Poland \\ Correspondence should be addressed to Ewa Pawluszewicz; e.pawluszewicz@pb.edu.pl
}

Received 7 April 2015; Revised 18 October 2015; Accepted 20 October 2015

Academic Editor: Alicia Cordero

Copyright (c) 2015 Ewa Pawluszewicz. This is an open access article distributed under the Creative Commons Attribution License, which permits unrestricted use, distribution, and reproduction in any medium, provided the original work is properly cited.

\begin{abstract}
The problem of controllability to a given convex target set of linear fractional systems with $h$-difference fractional operator of Caputo type is studied. Necessary and sufficient conditions of controllability with constrained controllers for such systems are given. Problem of approximation of a continuous-time system with Caputo fractional differential by a discrete-time system with $h$-difference fractional operator of Caputo type is discussed.
\end{abstract}

\section{Introduction}

In the control theory there are three fundamental concepts: controllability, observability, and stability of the given control system. Controllability means that there is a possibility to transfer the considered system from a given initial state to a final state using controls from some set. Observability means the possibility of the reconstruction of an initial state on the base of controls (inputs) and output sequences. It seems that these concepts in the classical linear control systems (for both continuous-time and discrete-time cases) are quite well investigated. In last years some of theirs extensions on nonuniform time domain have been done; see, for example, $[1,2]$. The other extension of the classical theory of control systems is introducing instead of the classical time derivative a fractional one (see, e.g., $[3,4]$ ) or instead of the classical difference operator a fractional difference (see, e.g., [5-7]). In the recent years the fractional calculus is viewed as a power tool in descriptions of real system' behaviours; see, for example, [8-10].

The controllability problem of fractional linear systems was studied, for example, in [3, 7, 11-14]. In [15] controllability of linear systems with Caputo type difference operators with two different fractional orders was investigated. Later on these results were extended to $h$-difference linear control systems with $n$ different fractional orders in [16].

In many cases it is assumed that the set of control values coincides directly with the whole control space, but in practise this set should be bounded; see, for example, [17]. A restriction on controls possesses some difficulties for controllability conditions. Necessary and sufficient conditions for controllability of classical linear systems with control constrains were proven in [18-20]. In [21] some of results of [20] were extended to any time model. Conditions for constrained controllability for a special class of linear systems with two fractional orders and with Caputo type difference operators were tackled in [22]. Generally, in the field of systems with fractional order, this topic is worthy of investigation. To the best of our knowledge, there exist only few works in continuous-time case; see [23].

The goal of the present work is to give conditions for possibility of steering of linear $h$-difference control system with fractional difference Caputo type operator to a given convex target set $X$ from a specified initial state. To this aim in Section 2 there are presented the needed definitions and facts from fractional $h$-difference calculus. Next, in Section 3 , using the separation hyperplane theorem, necessary and sufficient conditions for constrained controllability of the considered system are discussed. In [22] the first step in the investigation of the problem of constrained controllability for discrete-time fractional two-order linear systems, using the specific transition and gramian controllability matrices, was discussed. Now, in Section 3, using the $\mathscr{Z}$-transform method and Mittag-Leffler function introduced in [24] and similar reasoning (that idea in fact comes from [20]), necessary and sufficient conditions for constrained controllability of the 
considered system are presented. These results can be quite easily extended to multistep and/or multiorder fractional systems, but notation would be more complicated.

As real continuous-time models in some cases should be approximated by discrete-time models, the problem of approximation of a continuous fractional coast function by a discrete-time one is discussed in Section 4. Section 5 discussed a particular case of constrained controllability, namely, the case when the target set consists only of one point. This specific situation obtains when a given object is transferred to a predetermined position.

For our goal let us recall the separation theorem. Suppose that $X$ is a real normed space and $A, B \subset X$.

Theorem 1 (see [25]). Suppose that $A$ and $B$ are convex and disjoint sets. Let the interior of $A$ be nonempty. Then sets $A$ and $B$ are separable.

Theorem 1 implies that there exists a linear continuous functional $f$ such that

$$
\sup _{y \in B} f(y) \leq \inf _{x \in A} f(x)
$$

\section{Preliminaries}

Let us start from introduction of the basic notations and facts needed in the subsequent sections.

For $\alpha>0, h>0$, and $a \in \mathbb{R}$ let $(h \mathbb{N})_{a}:=\{a, a+$ $h, a+2 h, \ldots\}$. For a function $x:(h \mathbb{N})_{a} \rightarrow \mathbb{R}$, the forward $h$-difference operator is defined by

$$
\left(\Delta_{h} x\right)(t):=\frac{x(t+h)-x(t)}{h}, \quad t=a+k h, k \in \mathbb{N}_{0}
$$

and the $h$-difference sum is given by

$$
\begin{aligned}
& \left({ }_{a} \Delta_{h}^{-1} x\right)(t):=h \sum_{i=0}^{k} x(a+i h), \\
& \left({ }_{a} \Delta_{h}^{-1} x\right)(a):=0,
\end{aligned}
$$

where $t=a+(k+1) h$ and $k \in \mathbb{N}_{0}$.

Let

$$
\varphi_{\mu}(n)=\left(\begin{array}{c}
n+\mu-1 \\
n
\end{array}\right), \quad \text { for } n \in \mathbb{N}_{0},
$$

denotes the family of binomial sequences on $\mathbb{Z}$ parameterized by $\mu>0$. Recall that (see [24])

$$
\left(\varphi_{\mu} * \bar{x}\right)(n):=\sum_{s=0}^{n}\left(\begin{array}{c}
n-s+\mu-1 \\
n-s
\end{array}\right) \bar{x}(s)
$$

denotes the convolution of sequences $\varphi_{\mu}$ and $\bar{x}(s):=x(a+s h)$, $n \in \mathbb{N}_{0}$. Then, the fractional $h$-sum of order $\alpha>0$ for a real valued function $x$ defined on $(h \mathbb{N})_{a}$ is given by

$$
\left({ }_{a} \Delta_{h}^{-\alpha} x\right)(t):=h^{\alpha}\left(\varphi_{\alpha} * \bar{x}\right)(n),
$$

with $t=a+(\alpha+n) h$. Note that the operator ${ }_{a} \Delta_{h}^{-\alpha}$ maps real valued functions defined on the set $(h \mathbb{N})_{a}$ into real valued functions defined on the set $(h \mathbb{N})_{a+\alpha h}$.

Let the discrete Mittag-Leffler two-parameter function be defined as follows (see [24]):

$$
E_{(\alpha, \beta)}(\lambda, n):=\sum_{k=0}^{\infty} \lambda^{k} \varphi_{k \alpha+\beta}(n-q k) .
$$

Note that

$$
\begin{aligned}
& E_{(\alpha, \alpha)}(\lambda, n)=\sum_{k=0}^{\infty} \lambda^{k}\left(\begin{array}{c}
n-q k+(k+1) \alpha-1 \\
n-k
\end{array}\right), \\
& E_{(\alpha, 1)}(\lambda, n)=\sum_{k=0}^{\infty} \lambda^{k}\left(\begin{array}{c}
n-q k+k \alpha \\
n-k
\end{array}\right) .
\end{aligned}
$$

Definition 2 (see [26]). Let $\alpha \in(0,1]$ and $a \in \mathbb{R}$. The Caputo type fractional $h$-difference operator ${ }_{a} \Delta_{h, *}^{\alpha}$ of order $\alpha$ for a function $x:(h \mathbb{N})_{a} \rightarrow \mathbb{R}$ is defined by

$$
\left({ }_{a} \Delta_{h, *}^{\alpha} x\right)(t):=\left({ }_{a} \Delta_{h}^{-(1-\alpha)}\left(\Delta_{h} x\right)\right)(t),
$$

where $t \in(h \mathbb{N})_{a+(1-\alpha) h}$.

Note that for any $\alpha \in(0,1]$ the operator ${ }_{a} \Delta_{h, *}^{\alpha}$ changes the domain of the function $x$; that is, it maps real valued functions defined on $(h \mathbb{N})_{a}$ into real valued functions defined on $(h \mathbb{N})_{a+(1-\alpha) h}$. Additionally, for $\alpha=1$ it holds that $\left({ }_{a} \Delta_{h, *}^{1} x\right)(t)=\left(\Delta_{h} x\right)(t)$. We will need the backward shifted discrete Mittag-Leffler function, that is, function $E_{(\alpha, \alpha)}^{\rho}\left(A h^{\alpha}, n\right):=E_{(\alpha, \alpha)}\left(A h^{\alpha}, n-1\right)$.

\section{Linear Control Systems with Fractional Order}

Let us consider the following linear control system of the form

$$
\left({ }_{a} \Delta_{h, *}^{\alpha} x\right)(n h)=A x(n h+a)+B u(n h)
$$

with an initial condition $x(a)=x_{0}$ and $n \in \mathbb{N}_{0}$. Therin $x$ : $(h \mathbb{N})_{a} \rightarrow \mathbb{R}^{p}$ is a state vector, $A \in \mathbb{R}^{p \times p}, B \in \mathbb{R}^{m \times p}, a=$ $(\alpha-1) h$, and the values $u(n h)$ of control $u$ are elements of an arbitrary set $\Omega \subseteq \mathbb{R}^{m}$. Denote the state forward trajectory of system (10), that is, a solution which is uniquely defined by initial state $x_{0}$ and control $u \in \Omega$ by $\psi\left(\cdot, x_{0}, u\right)$.

Corollary 3. System (10) with initial condition $x(a)=x_{0}$ and a fixed control $u$ has the unique solution given by

$$
\begin{aligned}
\psi\left(s, x_{0}, u\right)= & E_{(\alpha, 1)}\left(A h^{\alpha}, n\right) x_{0} \\
& +\left(E_{(\alpha, \alpha)}^{\rho}\left(A h^{\alpha}, \cdot\right) * B \bar{u}\right)(n),
\end{aligned}
$$

where $\bar{u}(n)=h^{\alpha} u(n h)$.

Proof. The proof follows from the properties of $\mathscr{Z}$-transform of the fractional Caputo type difference operator and it mimics (with respect to $h$ ) the proof of the similar result with $h=1$ given in [24]. 
The set of all states that can be reached by system (10) from the initial state $x(a)=x_{0}$ in a finite number of steps $s$ is called a reachable set and denoted by $\mathscr{R}_{x_{0}}(s)$; see, for example, [27]; that is,

$$
\begin{aligned}
& \mathscr{R}_{x_{0}}(s) \\
& \quad:=\left\{x \in \mathbb{R}^{n}: x\left(t_{1}\right)=\psi\left(s, x_{0}, u\right), \text { for some } u \in \Omega\right\}
\end{aligned}
$$

and $\mathscr{R}_{x_{0}}(0):=\{0\}$. Similarly as in [28] one can show that if $\Omega \subset \mathbb{R}^{m}$ is compact and convex set, then $\mathscr{R}_{x_{0}}(s)$ is a compact and convex subset of $\mathbb{R}^{p}$ for any $x_{0}$.

Let $X \subset \mathbb{R}^{n}$ denote a target set and $\Omega \subset \mathbb{R}^{m}$.

Definition 4. System (10) is $\Omega$-controllable to $X$ in a finite number of steps $s$ from the initial state $x(a)=x_{0}$ if there exists a control $u \in \Omega$ which transfers this system to the set $X$ at a prescribed number of steps $s$.

Let $s \in \mathbb{N}_{0}$ and $\bar{u}(n):=h^{\alpha} u(n h)$. For a vector $\lambda \in \mathbb{R}^{p}$ let us define the cost function in the following way:

$$
\begin{aligned}
I\left(x_{0}, s, \lambda\right):= & \lambda^{T} E_{(\alpha, 1)}\left(A h^{\alpha}, s\right) x_{0} \\
& +\max _{u \in \Omega}\left[\left(E_{(\alpha, \alpha)}^{\rho}\left(A h^{\alpha}, \cdot\right) * B \bar{u}\right)(s)\right] \\
& -\inf _{x \in X} \lambda x^{T},
\end{aligned}
$$

where $\lambda^{T}$ denotes the transposition of the vector $\lambda \in \mathbb{R}^{p}$. From definition of the convolution it follows that (13) can be rewritten as

$$
\begin{aligned}
& I\left(x_{0}, s, \lambda\right) \\
& =\lambda^{T} E_{(\alpha, 1)}\left(A h^{\alpha}, s\right) x_{0} \\
& \quad+h^{\alpha} \max _{u \in \Omega}\left[\sum_{\nu=0}^{s-1} E_{(\alpha, \alpha)}\left(A h^{\alpha}, s-v-1\right) B(\nu h)\right] \\
& \quad-\inf _{x \in X} \lambda x^{T} .
\end{aligned}
$$

Note that if $\alpha=1$ and $h=1$, then

$$
\begin{aligned}
I\left(x_{0}, s, \lambda\right)= & \lambda^{T} E_{(1,1)}(A, s) x_{0} \\
& +\max _{u \in \Omega}\left[\sum_{\nu=0}^{s-1} E_{(1,1)}(A, s-v-1) B u(\nu)\right] \\
& -\inf _{x \in X} \lambda x^{T} .
\end{aligned}
$$

Theorem 5. System (10) is $\Omega$-controllable to an open convex target set $X$ in steps from the given initial state $x(a)=x_{0}$ if and only if there exists a vector $\lambda \in \mathbb{R}^{p}$ such that $I\left(x_{0}, s, \lambda\right)>0$.

Proof. The proof is similar to the proof of the respective results in [21].

“ $\Rightarrow$ " $\Omega$-controllability of system $(10)$ to the target set $X$ in $s$ steps from initial state $x(a)=x_{0}$ means that the set
$\mathscr{R}_{x_{0}}(s) \cap X$ is not empty. So, by contradiction, Theorem 1 and inequality (1) imply that

$$
\sup _{\psi\left(s, x_{0}, u\right) \in \mathscr{R}_{x_{0}}(s)} \lambda \psi^{T}\left(s, x_{0}, u\right)>\inf _{x \in X} \lambda x^{T}
$$

where $\psi^{T}$ is the transposition of the vector function $\psi$. Then

$$
\sup _{\psi\left(s, x_{0}, u\right) \in \mathscr{R}_{x_{0}}(s)} \lambda \psi^{T}\left(s, x_{0}, u\right)-\inf _{x \in X} \lambda x^{T}>0,
$$

so

$$
\begin{aligned}
& \sup _{\psi\left(s, x_{0}, u\right) \in \mathscr{R}_{x_{0}}\left(t_{1}\right)}\left[\lambda^{T} E_{(\alpha, 1)}\left(A h^{\alpha}, s\right) x_{0}\right. \\
& \left.+\left(E_{(\alpha, \alpha)}^{\rho}\left(A h^{\alpha}, \cdot\right) * B \bar{u}\right)(n)\right]-\inf _{x \in X} \lambda x^{T}>0 .
\end{aligned}
$$

Since $\Omega$ is compact, then inequality (18) can be rewritten as

$$
\begin{aligned}
& \lambda^{T} E_{(\alpha, 1)}\left(A h^{\alpha}, s\right) x_{0}+\max _{u \in \Omega}\left[E_{(\alpha, \alpha)}^{\rho}\left(A h^{\alpha}, \cdot\right) * B \bar{u}\right](n) \\
& -\inf _{x \in X} \lambda x^{T}>0 .
\end{aligned}
$$

Hence, there exists $\lambda \in \mathbb{R}^{p}$ such that $I\left(x_{0}, s, \lambda\right)>0$.

" $\Leftarrow$ " First let us note that if system (10) is not $\Omega$ controllable to an open and convex target set $X$ in $s$ steps from the given initial state $x(a)=x_{0}$, then $\mathscr{R}_{x_{0}}\left(t_{1}\right) \cap X=\emptyset$. So, Theorem 1 implies that

$$
\sup _{\psi\left(s, x_{0}, u\right) \in \mathscr{R}_{x_{0}}(s)} \lambda \psi^{T}\left(s, x_{0}, u\right) \leq \inf _{x \in X} \lambda x^{T} .
$$

On the other hand, if there exists a vector $\lambda \in \mathbb{R}^{p}$ such that $I\left(x_{0}, s, \lambda\right)>0$, then taking into account compactness of the set $\Omega$, the following holds:

$$
\begin{aligned}
I( & \left.x_{0}, s, \lambda\right) \\
= & \lambda^{T} E_{(\alpha, 1)}(A, s) x_{0} \\
& +\max _{u \in \Omega}\left[\left(E_{(\alpha, \alpha)}^{\rho}\left(A h^{\alpha}, \cdot\right) * B \bar{u}\right)(s)\right]-\inf _{x \in X} \lambda x^{T} \\
= & \sup _{u \in \Omega}\left[\lambda^{T} E_{(\alpha, 1)}(A, s) x_{0}+\left[E_{(\alpha, \alpha)}^{\rho}\left(A h^{\alpha}, \cdot\right) * B \bar{u}\right](s)\right] \\
& -\inf _{x \in X} \lambda x^{T}=\sup _{\psi\left(s, x_{0}, u\right) \in \mathscr{R}_{x_{0}}(s)} \lambda^{T} \psi\left(s, x_{0}, u\right)-\inf _{x \in X} \lambda x^{T} \\
> & 0 .
\end{aligned}
$$

So, we have contradiction with (20) and in the consequence with the assumption that system (10) is not $\Omega$-controllable to an open convex target set $X$ in the finite number of steps $s$ from the given initial state.

Corollary 6. If the target set $X$ is closed and convex, then system (10) is $\Omega$-controllable to $X$ from initial state $x(0)=x_{0}$ in a finite number of steps $s$ if and only if for any vector $\lambda \in \mathbb{R}^{p}$ such that $\|\lambda\|=1$ it holds that $\min _{\|\lambda\|=1} I\left(x_{0}, s, \lambda\right) \geq 0$. 
Proof. " $\Rightarrow$ " Theorem 5 implies that $I\left(x_{0}, s, \lambda\right)>0$, so also $\inf _{\lambda} I\left(x_{0}, s, \lambda\right) \geq 0$. Then $\min _{\|\lambda\|=1} I\left(x_{0}, s, \lambda\right) \geq 0$ for any $\lambda \in \mathbb{R}^{p}$.

“ $\Leftarrow$ " Inequality $\min _{\|\lambda\|=1} I\left(x_{0}, s, \lambda\right) \geq 0$ implies that also $\inf _{\lambda} I\left(x_{0}, s, \lambda\right) \geq 0$, so the thesis follows from Theorem 5 .

\section{Approximation of Continuous-Time Control Systems of Fractional Order}

Since during the study of real phenomena sometimes continuous-time models should be approximated by discretetime ones, in this section we consider the problem of approximation a coast function associated with a continuoustime linear fractional control system with Caputo differential by the respective coast function associated with the discretetime linear control system with $h$-difference fractional operator of Caputo type.

Before stating the main result of this section let us recall some facts and results about approximation of a continuoustime linear control system with the Caputo fractional differential by an $h$-difference linear control system with fractional Caputo type operator.

Proposition 7 (see [29]). Let $\alpha \in(0,1], h>0, a \in \mathbb{R}$, and $t \geq a$. If $\bar{t}_{h}:=a+(1-\alpha) h+n h$, where $n=[(t-a) / h]+1$ and $[(t-a) / h]$ is the greatest integer less than or equal to $(t-a) / h$, then $\lim _{h \rightarrow 0} \bar{t}_{h}=t$ and

$$
\lim _{h \rightarrow 0}\left(\bar{t}_{h}-a\right)_{h}^{(-\alpha)}=(t-a)^{-\alpha} .
$$

Let us recall that the Caputo fractional derivative of order $\alpha, 0<\alpha \leq 1$, of a real continuous function $x$ is defined as

$$
{ }_{a}^{C} D^{\alpha} x(t)=\frac{1}{\Gamma(n-\alpha)} \int_{a}^{1} \frac{x^{(n)}(\tau)}{(t-\tau)^{\alpha+1-n}} d \tau,
$$

where $\Gamma$ denotes the gamma function.

Proposition 8 (see [29]). Let $\alpha \in(0,1]$, let $h>0$, and let $f$ be a continuous real function with integrable $f^{\prime}$ defined on some interval $[a, T]$ with $T>a>0$, and $\bar{t}_{h}:=a+(1-\alpha) h+n h$, where $n=[(t-a) / h]+1$. Then

$$
\left({ }_{a}^{C} D^{\alpha} f\right)(t)=\lim _{h \rightarrow 0}\left({ }_{a} \Delta_{h, *}^{\alpha} f\right)\left(\bar{t}_{h}\right) .
$$

Consider an initial value problem defined for the Caputo fractional derivative with order $\alpha \in(0,1]$ of the form

$$
{ }_{a}^{C} D^{\alpha} x(t)=f(t, x(t)), \quad x(a)=x_{a} \in \mathbb{R}^{n},
$$

where $t \in(a, T], a>0, x:(a, T] \rightarrow \mathbb{R}^{p}$. Let $(a, T]_{(h \mathbb{N})_{a}}:=$ $(a, T] \cap(h \mathbb{N})_{a+(1-\alpha) h}$. Suppose that system (25) is uniformly sampled with a sampling step $h, h>0$. As a result of this operations, we can consider an initial value problem given for the $h$-difference fractional operator of Caputo type with order $\alpha \in(0,1]$ :

$$
\left({ }_{a} \Delta_{h, *}^{\alpha} y\right)(t)=f(t, y(t+a)), \quad y(a)=x(a)=x_{a},
$$

where $a=(\alpha-1) h, n \in \mathbb{N}_{0}, y:(a, T]_{(h \mathbb{N})_{a}} \rightarrow \mathbb{R}^{p}$.

Let $\bar{t}_{h}:=a+(1-\alpha) h+n h$ with $n=[(t-a) / h]+1$ and $a=(\alpha-1) h, \alpha \in(0,1]$. Also let $\bar{x}:(a, T]_{(h \mathbb{N})_{a}} \rightarrow \mathbb{R}^{n}$.
Proposition 9 (see [29]). The solution $x$ of system

$$
\left({ }_{0}^{C} D^{\alpha} x\right)(t)=f(t, x(t)), \quad x(0)=x_{0}
$$

is approximated by the solution of system

$$
\left({ }_{a} \Delta_{h, *}^{\alpha} \bar{x}\right)(t)=f(t, \bar{x}(t)), \quad \bar{x}(a)=x_{0},
$$

in values via the limit $\lim _{h \rightarrow 0} \bar{x}\left(t_{h}\right)=x(t)$.

Let us consider a continuous-time linear control system with the Caputo fractional differential

$$
\left({ }_{0}^{C} D^{\alpha} x\right)(t)=A x(t)+B u(t), \quad x(0)=x_{0},
$$

where $t \in(0, T], T>0, A \in \mathbb{R}^{p \times p}, B \in \mathbb{R}^{m \times p}$, and the values $u(t)$ of control $u$ are elements of an arbitrary set $\Omega \subseteq \mathbb{R}^{m}$. The trajectory $\gamma\left(t, x_{0}, u\right)$ of color blue system (29) is described by (see [11])

$$
\begin{aligned}
& \gamma\left(t, x_{0}, u\right) \\
& =\mathscr{E}_{\alpha}\left(A t^{\alpha}\right) x_{0} \\
& \quad+\int_{0}^{t}(t-s)^{\alpha-1} \mathscr{E}_{(\alpha, \alpha)}\left(A(t-s)^{\alpha}\right) B u(s) d s,
\end{aligned}
$$

where $\mathscr{E}_{(\alpha, \beta)}$ denotes two-parameter continuous MittagLeffler function defined as

$$
\mathscr{E}_{(\alpha, \beta)}(z):=\sum_{k=0}^{\infty} \frac{z^{k}}{\Gamma(k \alpha+\beta)}
$$

for $\alpha, \beta>0$ and $\mathscr{E}_{(\alpha)}(z):=\sum_{k=0}^{\infty}\left(A^{k} t^{k \alpha} / \Gamma(k \alpha+1)\right)$. Using the convolution operator formula (30) can be rewritten as

$$
\begin{aligned}
\gamma\left(t, 0, x_{0}, u\right)= & \mathscr{E}_{(\alpha)}\left(A t^{\alpha}\right) x_{0} \\
& +\left(t^{\alpha-1} \mathscr{E}_{(\alpha, \alpha)}\left(A t^{\alpha}\right) * B u\right)(s) .
\end{aligned}
$$

Similarly as in Section 3, we can define the reachable set for system (29), denoted by $\mathscr{R}_{x_{0}}(t)$, as the set of all states that can be reached by system (29) from the initial state $x(0)=x_{0}$ in a finite time $t \in(0, T]$; that is,

$$
\begin{aligned}
& \mathscr{R}_{x_{0}}(t):=\left\{x \in \mathbb{R}^{n}: x(t)\right. \\
& \left.\quad=\gamma\left(t, 0, x_{0}, u\right), \text { for some } u \in \Omega\right\} .
\end{aligned}
$$

By the same motivation as in [28] one can note that if $\Omega \subset \mathbb{R}^{m}$ is compact and convex set, then $\mathscr{R}_{x_{0}}(t)$ is also a compact and convex subset of $\mathbb{R}^{p}$ for any $x_{0}$.

Let $X \subset \mathbb{R}^{n}$ denote a target set and $\Omega \subset \mathbb{R}^{m}$.

Definition 10. System (29) is $\Omega$-controllable to $X$ in a time $t \epsilon$ $(0, T], T$-positive arbitrary but fixed, from initial state $x(0)=$ $x_{0}$ if there exists a control $u \in \Omega$ which transfers this system to the target set $X$ in time $t$. 
For a vector $\lambda \in \mathbb{R}^{p}$ let us define the cost function in the following way:

$$
\begin{aligned}
\widehat{I}\left(x_{0}, t, \lambda\right):= & \lambda^{T} \mathscr{E}_{(\alpha)}\left(A t^{\alpha}\right) x_{0} \\
& +\max _{u \in \Omega}\left[\left(t^{\alpha-1} \mathscr{E}_{(\alpha, \alpha)}\left(A t^{\alpha}\right) * B u\right)(s)\right] \\
& -\inf _{x \in X} \lambda x^{T}
\end{aligned}
$$

for any $t \in(0, T]$ with positive $T$ arbitrary but fixed.

Proposition 11. Let $\alpha \in(0,1], h>0, a \in \mathbb{R}$, and $t \geq a$. If $\bar{t}_{h}:=(1-\alpha) h+n h$, then the continuous Mittag-Leffler function $\mathscr{E}_{(\alpha, \alpha)}(A t)$ is approximated by the the discrete Mittag-Leffler two-parameter function $E_{(\alpha, \alpha)}\left(A, t_{h}\right)$.

Proof. By Proposition 9 the solution of IVP $\left({ }_{0}^{C} D^{\alpha} x\right)(t)=$ $A x(t), x(0)=x_{0}$, that is, by

$$
x(t)=\mathscr{E}_{(\alpha)}\left(A t^{\alpha}\right) x_{0}, \quad t \in(0, T],
$$

is approximated by the solution of system $\left({ }_{0} \Delta_{h, *}^{\alpha} \bar{x}\right)(t)=$ $A \bar{x}(t), \bar{x}(0)=x_{0}$, that is, by

$$
\bar{x}(t)=E_{(\alpha)}\left(A h^{\alpha}, t_{h}\right) x_{0}, \quad t_{h} \in(0, T]_{(h \mathbb{N})_{a}} .
$$

Additionally, from Proposition 7, it follows that $\lim _{h \rightarrow 0} \bar{t}_{h}=t$. Then $\mathscr{E}_{(\alpha)}\left(A t^{\alpha}\right)$ can be approximated by $E_{(\alpha)}\left(A h^{\alpha}, t_{h}\right)$.

Corollary 12. Let $\alpha \in(0,1], h>0$, and $t \geq a$. If $\bar{t}_{h}:=$ $(1-\alpha) h+n h$, then the continuous Mittag-Leffler function $\mathscr{E}_{(\alpha, \beta)}(A t)$ is approximated by the the discrete Mittag-Leffler function $E_{(\alpha, \beta)}\left(A, t_{h}\right)$.

Proof. Result is a consequence of definitions of two-parameter Mittag-Leffler functions in continuous- and discretecases, respectively, and of Propositions 11 and 7.

Theorem 13. Assume that linear control system (29) is approximated by control system of the form

$$
\left({ }_{a} \Delta_{h, *}^{\alpha} \bar{x}\right)(t)=A \bar{x}(t)+B \bar{u}(t), \quad \bar{x}(0)=x_{0}
$$

with $a=(\alpha-1) h, n \in \mathbb{N}_{0}, \bar{x}:(a, T]_{(h \mathbb{N})_{a}} \rightarrow \mathbb{R}^{n}$. Then the cost function $\widehat{I}\left(x_{0}, t, \lambda\right)$ described by (34) can be approximated by the cost function $I\left(x_{0}, s, \lambda\right)$ given by (13).

Proof. If system (37) is the one that we can obtaine under the uniform sampling with sampling step $h>0$ from continuoustime linear system (29), then by Corollary 12 the trajectory of (29) can be approximated by the respective trajectory of (37) in values via the $\operatorname{limit}_{h \rightarrow 0} \bar{x}\left(t_{h}\right)=x(t)$. Hence thesis is a simple consequence of definition of the coast function (34), Corollary 12, and Proposition 11.

\section{Some Remarks}

Let us, using the classical reasoning (see, e.g., in [30, 31]), shortly remark a special case of constrained controllability of system (10) to the target set $X$ that consists only of one point.
Let us consider system (10) and let $X=\{0\}$. By Corollary 3 , the final state $x_{f}$ that can be obtained in a finite number of steps $s$ from the initial state $x(a)=x_{0}$ using controls $u$ with values in $\Omega \subset \mathbb{R}^{m}$ is given by

$$
\begin{aligned}
x_{f} & =\psi\left(s, x_{0}, u\right) \\
& =E_{(\alpha, 1)}\left(A h^{\alpha}, n\right) x_{0}+\left(E_{(\alpha, \alpha)}^{\rho}\left(A h^{\alpha}, \cdot\right) * B \bar{u}\right)(n)
\end{aligned}
$$

with $\bar{u}(n)=h^{\alpha} u(n h)$. Note that in fact

$$
\begin{aligned}
& \left(E_{(\alpha, \alpha)}^{\rho}\left(A h^{\alpha}, \cdot\right) * B \bar{u}\right)(n) \\
& \quad=h^{\alpha} \sum_{\nu=0}^{s-1} E_{(\alpha, \alpha)}\left(A h^{\alpha}, s-\nu-1\right) B u(\nu h) .
\end{aligned}
$$

Denoting by

$$
Q(s)=\sum_{k=0}^{s-1} E_{(\alpha, \alpha)}\left(A h^{\alpha}, s-k-1\right) B
$$

it can be easy to see that the target set $X=\{0\}$ to which system (10) should be steered from the given initial state $x(a)=x_{0}$ using controls $u_{s}=(u(0), u(h), \ldots, u((s-1) h)), u(k h) \in \Omega$, is given by

$$
0=E_{(\alpha, 1)}\left(A h^{\alpha}, s\right) x_{0}+h^{\alpha} Q(s) u_{s}
$$

Corollary 14. Let $0 \in \operatorname{int} \Omega$. Assume that system (10) is controllable in a finite number steps s. Then there exists a neighborhood $V_{0}$ of state $x(a)=x_{0} \in \mathbb{R}^{p}$ such that all elements of $V_{0}$ can be steered to set $X=\{0\}$ using the control $u_{s}$ with controls values in $\Omega$.

Proof. First of all note that system (10) is controllable if and only if matrix $Q(s)$ is invertible. So the sequence of controls

$$
u_{s}=-h^{-\alpha} Q^{-1}(s) E_{(\alpha, 1)}\left(A h^{\alpha}, s\right) x_{0}
$$

transfers the given initial state to the final state $x_{f}=0$, that is, to the target set $X=\{0\}$. Then there exists $K$ such that $\|\bar{u}(t)\| \leq K\|x\|$ for $t \in\{0, s, \ldots, s h\}$ and $x \in \mathbb{R}^{p}$, hence the thesis.

Corollaries 12 and 14 imply the following.

Corollary 15. If controllable system (29) with $t \in(a, T]$, $T>0$, is approximated by controllable (in finite number steps) system (37) with $t \in(a, T]_{(h \mathbb{N})_{a}}$ and $\tilde{u}=(u((1-\alpha) h), u((2-$ $\alpha) h), \ldots, u((s-\alpha) h))$, then there exists a neighborhood $V_{0}$ of state $x(0)=x_{0} \in \mathbb{R}^{p}$ such that all elements of $V_{0}$ can be steered to set $X=\{0\}$ using the control $u_{s}$ with controls values in $\Omega$.

\section{Conclusions}

In the paper we consider the problem of steering in a finite number of steps of a linear $h$-difference control system with Caputo type fractional operator to a given target set when the control is subject to specified magnitude constraints. Necessary and sufficient conditions for the constrained 
controllability for this class of systems to the target set were proposed. Since in some cases continuous-time models should be approximated by discrete-time ones, conditions for approximation of the coast function associated with a continuous-time linear fractional control system with Caputo differential by coast function associated with the respective (i.e., obtained by uniform sampling) discrete-time linear control system with $h$-difference fractional operator of Caputo type were presented.

\section{Conflict of Interests}

The author declares that there is no conflict of interests regarding the publication of this paper.

\section{Acknowledgments}

The project was supported by the founds of National Science Center granted on the bases of the decision number DEC2011/03/B/ST7/03476. The work was supported by Bialystok University of Technology grant G/WM/3/2012.

\section{References}

[1] Z. Bartosiewicz and E. Pawłuszewicz, "Realization of linear control systems on time scales," Control \& Cybernetics, vol. 35, no. 4, pp. 769-786, 2006.

[2] J. M. Davis, I. A. Gravagne, B. J. Jackson, and I. Marks, "Controllability, observability, realizability, and stability of dynamic linear systems," Electronic Journal of Differential Equations, vol. 37, pp. 1-32, 2009.

[3] T. Kaczorek, Selected Problems of Fractional Systems Theory, vol. 411 of Lecture Notes in Control and Information Sciences, Springer, Berlin, Germany, 2011.

[4] I. Podlubny, Fractional Differential Equations, Academic Press, San Diego, Calif, USA, 1999.

[5] T. Abdeljawad, "On Riemann and Caputo fractional differences," Computers \& Mathematics with Applications, vol. 62, no. 3, pp. 1602-1611, 2011.

[6] K. S. Miller and B. Ross, "Fractional difference calculus," in Proceedings of the International Symposium on Univalent Functions, Fractional Calculus and Their Applications, pp. 139152, Nihon University, Kōriyama, Japan, 1988.

[7] M. D. Ortigueira, "Fractional discrete-time linear systems," in Proceedings of the IEEE International Conference on Acoustics, Speech, and Signal Processing (ICASSP '97), vol. 3, pp. 2241-2244, Munich, Germany, April 1997.

[8] R. Hilfer, Applications of Fractional Calculus in Physics, World Scientific, Singapore, 2000.

[9] D. S. Karanjkar, S. Chatterij, and P. R. Vankateswam, "Trends in fractional order controller," International Journal of Emerging Technology and Advanced Engineering, vol. 2, no. 3, pp. 383-389, 2012.

[10] D. Sierociuk, A. Dzielinski, G. Sarwas, I. Petras, I. Podlubny, and T. Skovranek, "Modelling heat transfer in heterogeneous media using fractional calculus," Philosophical Transactions of the Royal Society A: Mathematical, Physical and Engineering Sciences, vol. 371, no. 1990, 2013.

[11] K. Balachandran and J. Kokila, "On the controllability of fractional dynamical systems," International Journal of Applied
Mathematics and Computer Science, vol. 22, no. 3, pp. 523-531, 2012.

[12] M. Bettayeb and S. Djennoune, "A note on the controllability and the obseravbility of fractional dyanmical systems," in Proceedings of the 2nd IFAC Workshop on Fractional Differentiation and Its Application, pp. 493-498, Porto, Portugal, July 2006.

[13] J. Klamka, "Local controllability of fractional discrete-time nonlinear systems with delay in control," in Advances in Control Theory, M. Buslowicz and K. Malinowski, Eds., pp. 25-34, Committe on Automatic Control and Robotics, Polish Academy of Sciences, Białystok, Poland, 2012.

[14] D. Mozyrska and E. Pawluszewicz, "Fractional discrete-time linear control systems with initialisation," International Journal of Control, vol. 85, no. 2, pp. 213-219, 2012.

[15] D. Mozyrska and E. Pawluszewicz, "Controllability of $h$ difference linear control systems with two fractional orders," International Journal of Systems Science, vol. 46, no. 4, pp. 662669, 2015.

[16] D. Mozyrska, E. Pawłuszewicz, and M. Wyrwas, "The $h$ difference approach to controllability and observability of fractional linear systems with Caputo type opeartor," Asian Journal of Control, vol. 17, no. 4, pp. 1163-1173, 2015.

[17] D. L. Abel, "Constrains vs controls," The Open Cybernetics \& Systemics Journal, vol. 4, pp. 14-27, 2010.

[18] B. R. Barmish and W. E. Schmitendorf, "A necessary and sufficient condition for local constrained controllability of a linear system," IEEE Transactions on Automatic Control, vol. 25, no. 1, pp. 97-100, 1980.

[19] W. E. Schmitendorf and W. G. Hwang, "Global reachability results for systems with constrained controllers," Journal of Optimization Theory and Applications, vol. 46, no. 4, pp. 581590, 1985.

[20] R. P. Van Til and W. E. Schmitendorf, "Constrained controllability of discrete-time systems," International Journal of Control, vol. 43, no. 3, pp. 941-956, 1986.

[21] K. Janglajew and E. Pawłuszewicz, "Constrained local controllability of dynamic systems on time scales," Advances in Difference Equations, vol. 2015, article 89, 2015.

[22] E. Pawłuszewicz and D. Mozyrska, "Constrained controllability of $h$-difference linear systems with two fractional orders," in Advances in the Theory and Applications of Non-Integer Order Systems, W. Mitkowski, J. Kacprzyk, and J. Baranowski, Eds., vol. 257 of Lecture Notes in Electrical Engineering, pp. 67-75, Springer, Berlin, Germany, 2013.

[23] K. Balachandran and J. Kokila, "Constrained controllability of fractional dynamical systems," Numerical Functional Analysis and Optimization, vol. 34, no. 11, pp. 1187-1205, 2013.

[24] D. Mozyrska and M. Wyrwas, "The $\mathscr{Z}$-transform method and delta type fractional difference operators," Discrete Dynamics in Nature and Society, vol. 2015, Article ID 852734, 12 pages, 2015.

[25] S. Rolewicz, Functional Theory and Control Theory, WNT, 1997 (Polish).

[26] D. Mozyrska and E. Girejko, "Overview of the fractional hdifference operators," in Advances in Harmonic Analysis and Operator Theory: The Stefan Samko Anniversary Volume, vol. 229 of Operator Theory: Advances and Applications, pp. 253-267, Springer, 2013.

[27] D. Mozyrska and E. Pawluszewicz, "Local controllability of nonlinear discrete-time fractional order systems," Bulletin of the Polish Academy of Sciences: Technical Sciences, vol. 61, no. 1, pp. 251-256, 2013. 
[28] E. D. Sontag, Mathematical Control Theory, Springer, 1998.

[29] D. Mozyrska, E. Girejko, and M. Wyrwas, "Fractional nonlinear systems with sequential operators," Central European Journal of Physics, vol. 11, no. 10, pp. 1295-1303, 2013.

[30] J. Zabczyk, Mathematical Control Theory: An Introduction, Birkhuser, Boston, Mass, USA, 1992.

[31] E. Pawłuszewicz, "Null-controllability of linear systems on time scales," Acta Mechanica et Automatica, vol. 6, no. 4, pp. 50-55, 2012. 


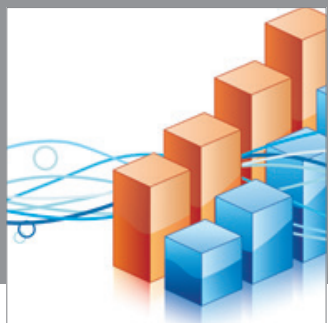

Advances in

Operations Research

mansans

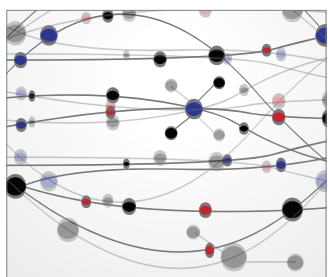

The Scientific World Journal
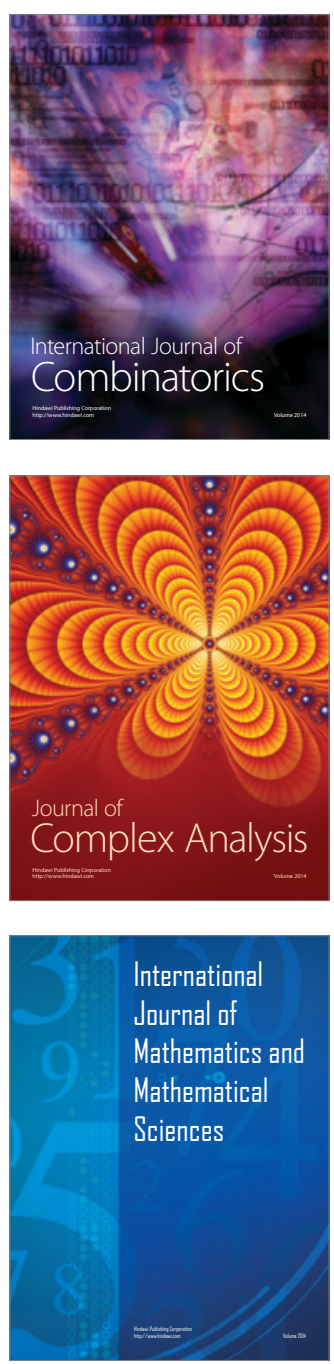
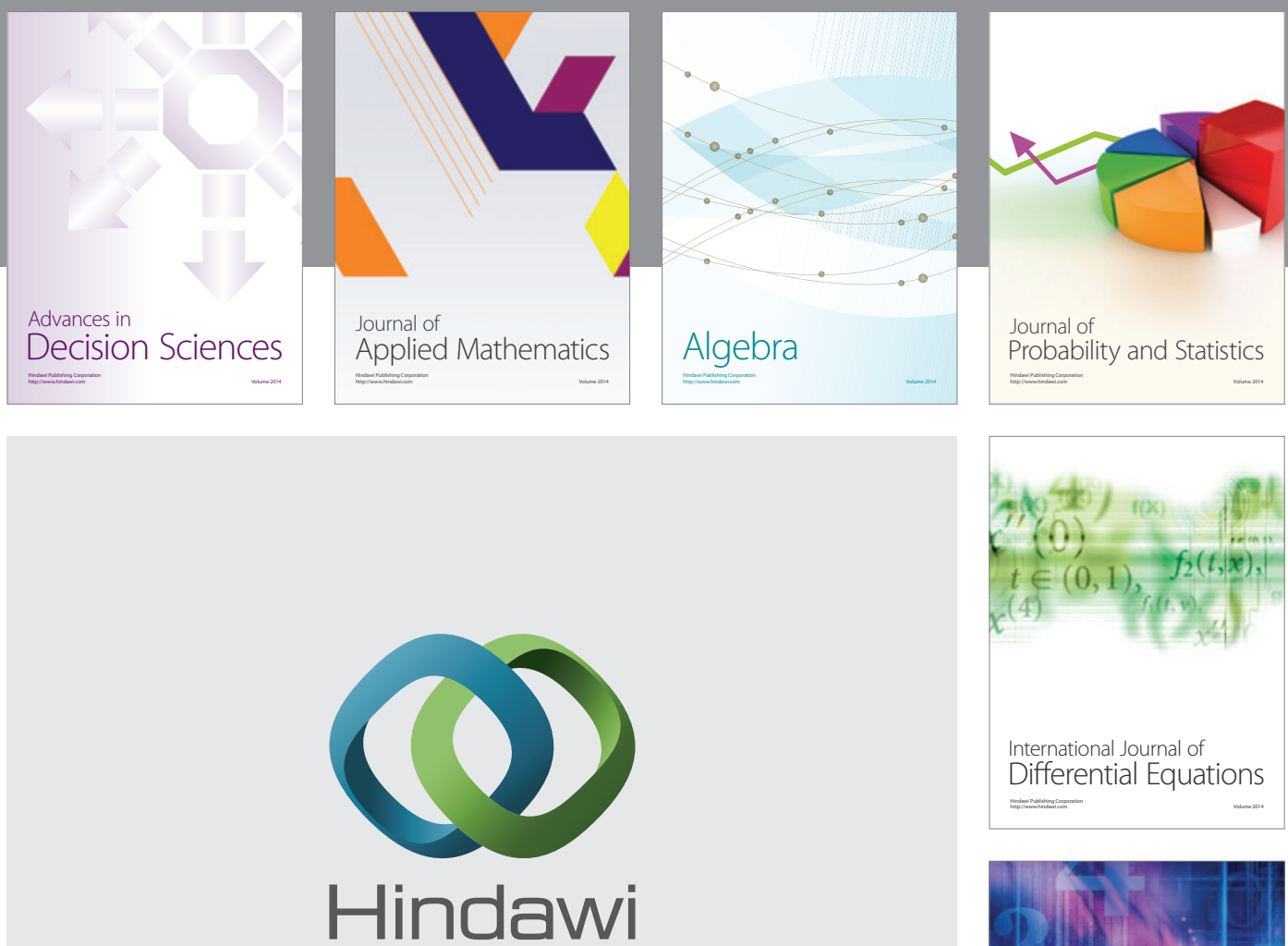

Submit your manuscripts at http://www.hindawi.com
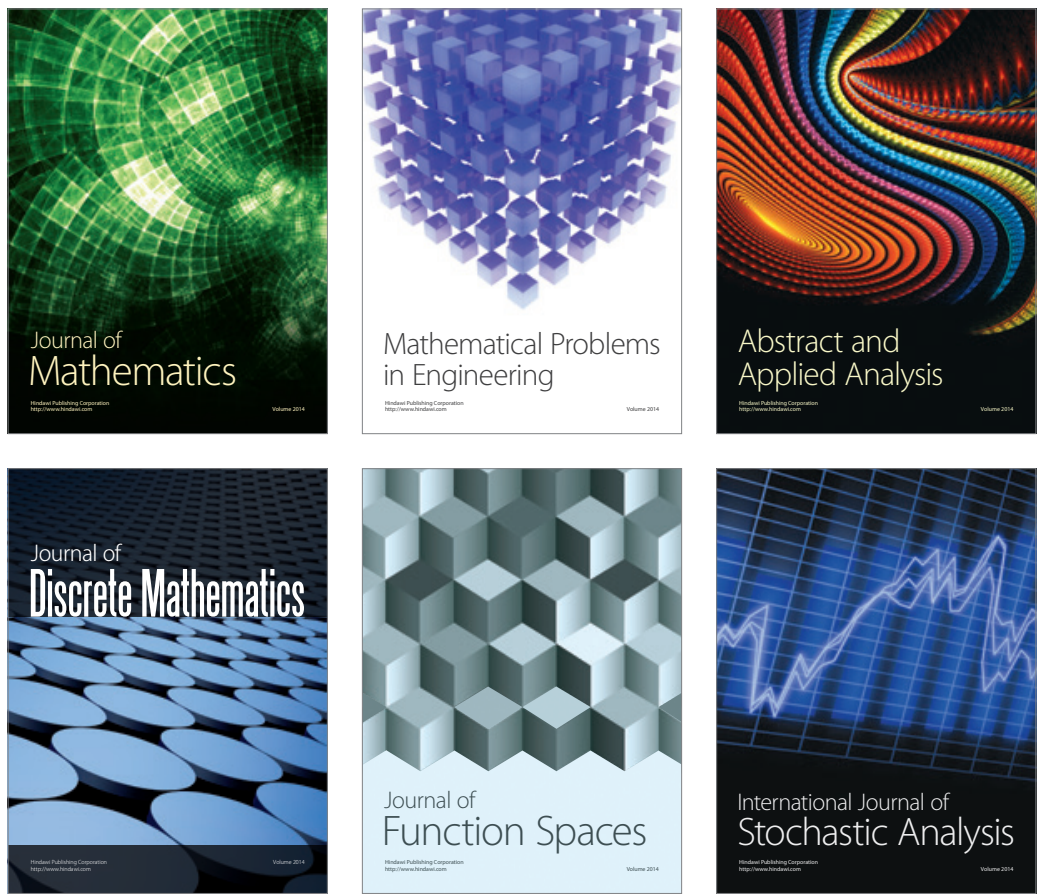

Journal of

Function Spaces

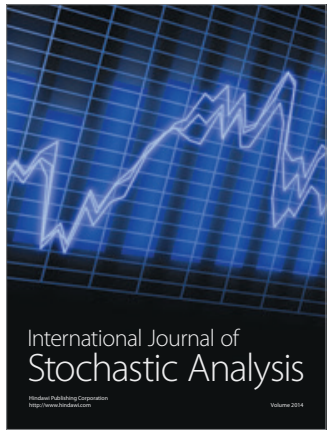

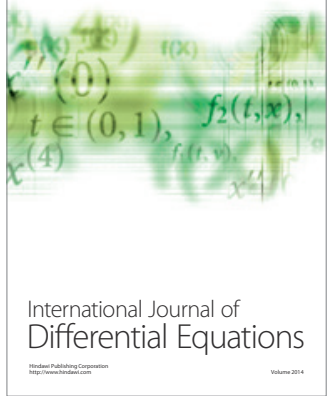
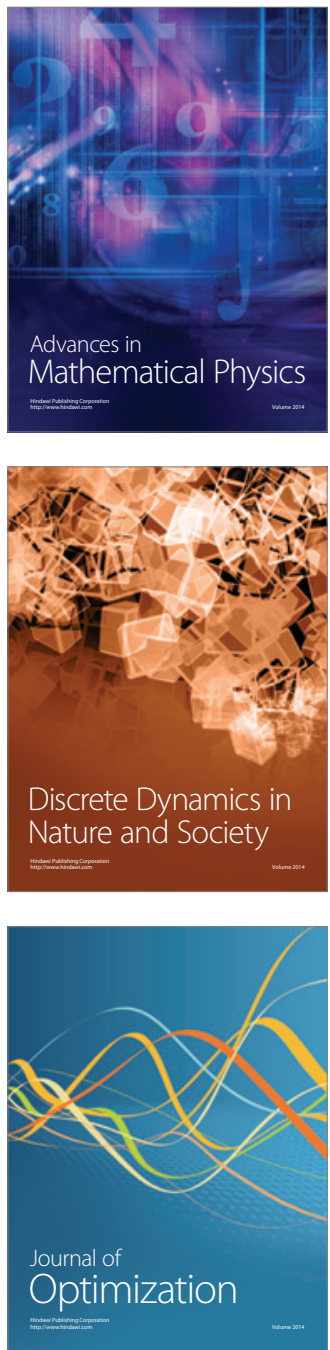